\title{
Optimum Power Allocation in Amplify and Forward Relay Selection Systems By Using ANNs
}

\author{
Ahmet EMIR ${ }^{1}$, Hakan KAYA ${ }^{2}$, Okan ERKAYMAZ ${ }^{3}$, Ertan ÖZTÜRK ${ }^{4}$
}

\begin{abstract}
Relay selection scheme in cooperative communication systems has been known as an effective technique that provides full diversity with efficient use of bandwidth in literature. Generally, the total power is shared equally between the source and the selected relay in relay selection schemes. However, the power allocation between the source and the selected relay is critical to further improve the error performance. In this study, we utilize Artificial Neural Networks (ANNs) to determine the optimum power allocation in Amplified-and Forward (AF) relay selection schemes for a minimum Bit Error Probability (BEP) performance over various channel gains. Since the analytical solution for an optimum power allocation in relay selection schemes is very complicated, the use of ANNs is an efficient and quick solution to further improve the performance.
\end{abstract}

Keywords - ANNs, Cooperative Diversity, Amplify and Forward Relay Selection, Optimum Power Allocation.

\section{INTRODUCTION}

A rtificial Neural Networks (ANNs) have been investigated by scientists over the past several decades. Because of the fast parallel processing and learning abilities, ANNs have been used as a computational method to solve nonlinear problems [1]. ANNs have been applied to different technologies such as image processing, automation, communication and signal processing [2] [3]. Neuron network topologies and number of neurons in ANNs change based on applications. Therefore, in order to achieve desired solutions specified to applications, different ANNs may be used. ANNs can obtain approximate solutions to theoretical and/or numeric problems that could be solved hardly theoretically and/or numerically.

In literature, there are some studies that present the applications of ANNs to wireless communications, such that ANNs are utilized in channel estimation between receiver and transmitter [4] [5]. Besides, ANNs has been shown as an effective technique that can be used to design channel

Ahmet EMIR $^{1}$ is with the Electrical Engineering Department, Bulent Ecevit University, Zonguldak, Turkey(e-mail:ahmet.emir@beun.edu.tr ).

Hakan KAYA ${ }^{2}$ is with the Electrical Engineering Department, Bulent Ecevit University, Zonguldak, Turkey(e-mail:hakan.kaya@beun.edu.tr ).

Okan ERKAYMAZ ${ }^{3}$ is with the Computer Engineering Department, Bulent Ecevit University, Zonguldak, Turkey (email:okan.erkaymaz@beun.edu.tr)

Ertan ÖZTÜRK ${ }^{4}$ is with the Electrical Engineering Department, Bulent Ecevit University, Zonguldak, Turkey(e-mail:eozturk@beun.edu.tr ). equalizer to remove non-linear distortion in wireless communication channels [6]. ANN is also used in Orthogonal Frequency Division Multiplexing (OFDM) systems in order to implement an adaptive modulation of the subcarriers. In [7], the inputs of ANN are the channel gain of all subcarriers, and the outputs of ANN are the modulation of all subcarriers.

Various channel factors such as interference, multi-path fading, path loss etc. may degrade the performance of wireless communication systems. In order to improve the performance, various diversity techniques can be used: Space diversity is one of the frequently used diversity techniques and realized by employing multiple antennas which are physically separated from one another. In this technique, the independent copies of data are transmitted/ received by more than one antenna to achieve different fadings for the independent copies of data. Therefore, the possibility of degradation of the transmitted data is reduced. The multi antenna systems are also called as Multi Input Multi Output (MIMO) systems [8]. On the other hand, in order to obtain space diversity, the distance between antennas in a MIMO system should be chosen at least the half of the wavelength of the transmitted signal [8]. Hence, the space diversity is not easy to implement in the uplink of a communication system, due to the physical limitations of a mobile user. To overcome this dilemma, mobile users cooperate with each other to achieve diversity through uplink. This method is called as cooperative communication (CC) in the literature [9], and the diversity achieved by a $\mathrm{CC}$ scheme is called cooperative diversity [9].

By using cooperative diversity, a user transmits both its own data and the data of other users in cooperation. A mobile user that helps the communication of another user is called as a relay. In cooperative diversity, in order to improve the quality of the communication link, the data should be processed at the relay. There are two methods defined in the literature [10] to process the data in a relay: Amplify and Forward (AF) and decode and forward (DF) methods. In AF method, the received data is amplified by the relay then this cooperative relay retransmits the amplified version of data to a destination. On the other hand, in DF method, the relay first decodes the received data, then forwards to a destination. In a $\mathrm{CC}$ system, the source may transmit the signal to the destination not only through a single relay but also through multi relays to increase diversity [11]. By using the multirelays, the diversity gain becomes as many as the number of 
relays in cooperation. Moreover, instead of using all available relays to cooperate, only one selected relay among the multirelays can be employed in order to achieve the same diversity gain of the multi-relay scheme [11]. In such scheme, the selected relay has the highest link qualities in terms of the transmission path, so called best relay.

In some cases the best relay may not be available to transmit, hence the second best or another one that is available can be used for relaying. Nevertheless, the performance of AF Cooperative Diversity with an Nth best relay is also presented in [12]. However, the total transmitted power is equally allocated at the source and the selected relay in [11-12]. On the other hand, the power allocation between the source and the selected best relay also affects the error performance of the cooperative systems.

In this study, we investigate the effects of the power allocation between the source and the selected relay to further minimize the error performance of an AF selection scheme. The contribution of this work is the use of ANN models to determine optimum power allocations in the considered system and compare the results of ANNs with those obtained numerically, under different channel link qualities.

The rest of this paper is organized as follows. In Section II, ANN methods are described. In Section III the considered CC System model is described and the selection scheme is presented. In Section IV presents the numerical results. Finally, conclusions are given in Section V.

\section{ANN METHODS}

ANNs process the parallel input data to produce proper outputs. ANNs perform the processes without any formulation between the inputs and the outputs. There are several parts for an artificial neuron such as input(s) with weight(s), activation function(s) and output(s). The weights are adjusted to achieve desired output. If an output is not good as possible, the process continuous until the desired output is generated for a given input with minimum error criteria. ANNs are so effective that they can solve complex equations very fast, and ANNs are easy tool to handle complex mathematic operations. ANNs are widely used for function approximation, clustering, classification and regression algorithms [13].

Different types of topology are present for ANNs. Most popular is Multi-Layer Perceptron (MLP) based on feed neural network. MLP model has at least three layers: input layer, one hidden layer and output layer [13]. According to different problems more hidden layer may be present on MLP Model. Computation algorithms are planned in these layers and the information is sent through layer by layer. Especially, the information is weighted between layer connections. MLP is chosen in this study, because of its efficient learning capacity and its ability to process large data.

ANN can be trained by using different learning algorithms. On the basis of these learning algorithms, weights of network are updated until the error between the desired output and the network output reduces to a minimum value. The error between the desired output and the network's output is computed as:
$\frac{1}{\mathrm{~N}} \sum_{\mathrm{k}=1}^{\mathrm{N}} \mathrm{d}_{\mathrm{k}}$

where $\mathrm{N}$ is the number of the output points, $\mathrm{d}_{\mathrm{k}}$ is kth desired output, $\mathrm{y}_{\mathrm{k}}$ is the $\mathrm{k}_{\mathrm{th}}$ output of network and defined as:

$y_{i}=f\left(\sum_{k=1}^{N} w_{i j} x_{i}\right)$

where $f($.$) is an activation function. The parameters \mathrm{w}_{\mathrm{ij}}$ are weight values and $x_{i}$ is $i_{\text {th }}$ input data

In this study, we utilize Levenberg-Marquardt backpropagation algorithm for trainings. MLP have five inputs and a single output point. MLP model have one hidden layer. MLP are trained and tested for different number of hidden neurons. In Fig 1 we show mean squared error (MSE) training performance versus the number of hidden neurons.

We obtain that the MLP topology having 12 hidden neurons demonstrates the best performance. Therefore, we utilize the architecture with 12 hidden neurons (5-12-1).

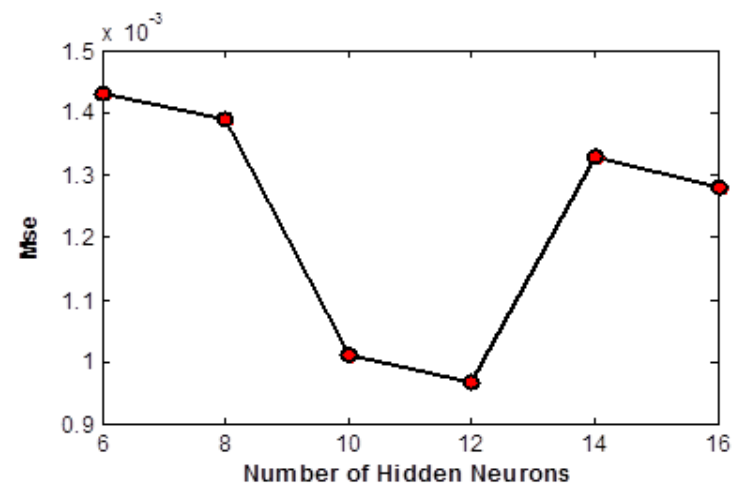

Fig. 1: MSE of MLP Architectures depends on number of hidden neurons

\section{III.AF RELAY SELECTION SCHEME}

In a CC system shown in Fig. 2, the source (S) transmits signal not only by direct link to destination (D) but also transmits through the best relay $\left(\mathrm{R}_{\text {sel }}\right)$ selected among multirelays in order to utilize selection diversity. The relay selection is performed by considering source-relay-destination cascaded link qualities of all available relays, then taking the one relay having the highest link qualities. After determining the best relay, the destination informs all relays about which relay is selected to transmit as the best relay through a reverse broadcast channel then other unselected relays turn to be idle.

In this study, cooperation strategy is carried out in two orthogonal phases to avoid interference between the two phases. In the first phase, the source broadcasts the information to destination and also all the relays at the same time. In the second phase, the best relay scales the received information (to equalize the effect of the channel fade between the source and the relay) and retransmits the amplified version of data to the destination. The destination combines the two received signals from the source in first phase and from the 
relay in second phase by using maximum-ratio combining (MRC) to maximize the overall SNR at the destination.

In the first phase, the signal transmitted from the source $s$ is received at both the destination and the $\mathrm{i}_{\mathrm{th}}$ relay as:

$$
\begin{gathered}
y_{S, D}=\sqrt{P \xi} h_{S, D} s+n_{S, D} \\
y_{S, R_{i}}=\sqrt{\mathrm{P}(1-\xi)} h_{S, R_{i}} s+n_{S, R i}
\end{gathered}
$$

where $\mathrm{P}, \mathrm{P}_{\mathrm{S}}$ and $\mathrm{P}_{\mathrm{Ri}}$ are the total power, the transmitted power at the source and at the $i_{\text {th }}$ relay, respectively. $\xi$ is the ratio of the transmitted power $\mathrm{P}_{\mathrm{S}}$ at the source over the total power $\mathrm{P}\left(\mathrm{P}_{\mathrm{S}}=\mathrm{P} . \xi\right)$, so called power ratio. Total transmitted power is defined as $\mathrm{P}=\mathrm{P}_{\mathrm{S}}+\mathrm{P}_{\mathrm{Ri}} \cdot \mathrm{h}_{\mathrm{SD}}$ and $\mathrm{h}_{\mathrm{SRi}}$ are the channel coefficients from the source to the destination and ith relay, respectively and they are modeled as Rayleigh flat fading channels with variance of $\sigma_{S, D}^{2}$ and $\sigma_{S, R_{i}}^{2}$, respectively. The terms $n_{S D}$ and $n_{S R i}$ present independently and identically distributed (i.i.d.) zero mean Gaussian noises with two sided power spectral density of $\mathrm{N}_{0} / 2$ per dimension.

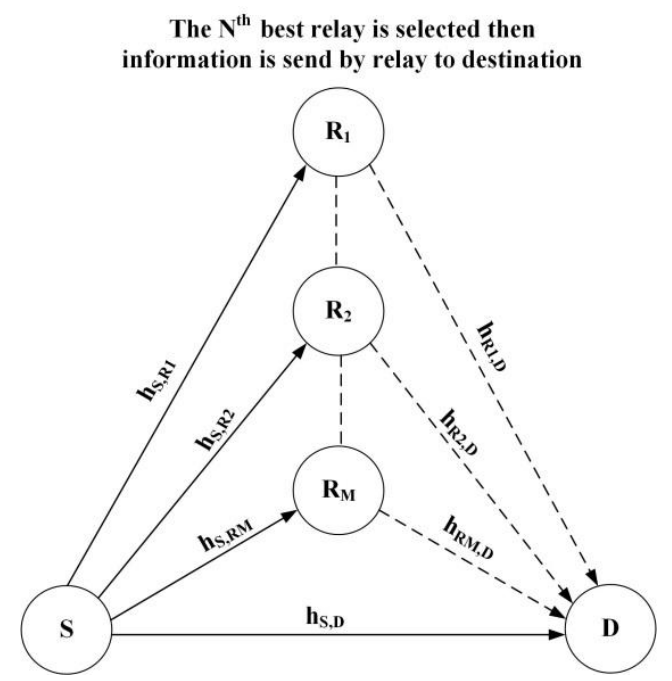

Fig. 2 Relay Selection Model

In the second phase, the best relay amplifies the signal from the source and forwards it to the destination via indirect link. The received signal at the destination in phase 2 through the best relay is given as

$$
\mathrm{y}_{\mathrm{R}_{\mathrm{sel}, \mathrm{D}}}=\beta \mathrm{h}_{\mathrm{R}_{\mathrm{se}, \mathrm{D}} \mathrm{D}} \mathrm{y}_{\mathrm{S}, \mathrm{R}_{\mathrm{sel}}}+\mathrm{n}_{\mathrm{R}_{\mathrm{sel}, \mathrm{D}}}
$$

where $h_{R_{s e l}, D}$ is the channel coefficient from the best relay to the destination and is modeled as Rayleigh flat fading channels with variance of $\sigma_{R_{s e l}, D}^{2}$. The terms nRD zero mean Gaussian noises with two sided power spectral density of $\mathrm{NO} / 2$ per dimension. $\beta$ is the amplifier gain and expressed as:

$\beta=\frac{\sqrt{P(1-\xi)}}{\sqrt{P \xi\left|h_{S, R_{S e l}}\right|^{2}+N_{0}}}$
The destination receives two copies of the signal $s$ through the source link and best relay link. With knowledge of the all channel coefficients, the total SNR at the destination can be written as:

$\gamma_{t o t}=\gamma_{S, D}+\max _{i \in M}\left\{\frac{\gamma_{S, R_{i}} \gamma_{R_{i}, D}}{\gamma_{S, R_{i}}+\gamma_{R_{i}, D}+1}\right\}$

In (6) the first component is the instantaneous SNR between S-D and given as $\gamma_{S, D}=h_{S, D}^{2} P_{S} / N_{0} \cdot \gamma_{S, R_{i}}=$ $h_{S, R_{i}}^{2} P_{S} / N_{0}$ is the instantaneous SNR of the source signal at $R_{i}$ and $\gamma_{R_{i}, D}=h_{R i, D}^{2} P_{S} / N_{0}$ is the instantenous SNR of the ith relay ( by $R_{i}$ ) at $D$. In (6), the second component is the end to end SNR, which is received at the destination from source through the best relay, is the harmonic mean of two exponential random variables which are related to the indirect link $\left(\gamma_{\mathrm{S}, \mathrm{Ri}, \mathrm{D}}\right)$.it is very complex to get the statistics of the harmonic mean of two random variables. So, the second component, given in the (6), should be expressed in more simple form. To simply the $\gamma_{S, R i, D}=\gamma_{S, R_{i}} \gamma_{R_{i}, D} /\left(\gamma_{S, R_{i}}+\gamma_{R_{i}, D}+1\right)$, a tight upper bound is obtained [12] as:

$\gamma_{S, R i, D} \leq \gamma_{i}=\min \left(\gamma_{S, R i}, \gamma_{R_{i}, D}\right)$

Using the upper bound $\left(\gamma_{i}\right)$, we can write an upper bound of the total SNR at the destination as

$\gamma_{t o t} \leq \gamma_{S, D}+\gamma_{\mathrm{S}, \mathrm{Rsel}, \mathrm{D}}=\gamma_{u b}$

where $\gamma_{\mathrm{S}, \mathrm{Rsel}, \mathrm{D}}=\max _{i \in M}\left\{\min \left(\gamma_{S, R i}, \gamma_{R_{i}, D}\right)\right\}$ is the instantaneous upper bounded SNR of the cascaded link from the source to the destination through the best relay

The average error probability in a flat fading channel is computed by averaging the conditional error probability over the probability density function (PDF) of the instantaneous total SNR $\left(\gamma_{\text {tot }}\right)$. Mathematically, $P_{b}(e)$ can be written as

$P_{b}(e)=\int_{0}^{\infty} P_{b}\left(e \mid \gamma_{t o t}\right) \times f_{\gamma_{t o t}}\left(\gamma_{t o t}\right) d_{\gamma_{t o t}}$

where $f_{\gamma_{t o t}}\left(\gamma_{t o t}\right)$ is the PDF of the total SNR at the destination given in (9). By assuming $\gamma_{S, D}$ and $\gamma_{\mathrm{S}, \mathrm{Rsel}, \mathrm{D}}$ are i.i.d. random and the modulation is Binary Phase Shift Keying (BPSK), $P_{b}(e)$ is rewritten in terms of moment generating function (MGF) defined $M_{\gamma}(s)=\int_{0}^{\infty} f_{\gamma}(\gamma) e^{-s \gamma} d \gamma \quad$ as following

$\overline{\mathrm{P}}_{\mathrm{b}}=\frac{1}{\pi} \int_{0}^{\pi / 2} \mathrm{M}_{\gamma_{\mathrm{S}, \mathrm{D}}}\left(\frac{1}{\sin ^{2}(\theta)}\right) \mathrm{M}_{\gamma_{\mathrm{Rsel}}}\left(\frac{1}{\sin ^{2}(\theta)}\right) \mathrm{d} \theta$

Where 
$M_{\gamma_{S, D}}(s)=\left(\frac{1}{1+s \bar{\gamma}_{S, D}}\right)$

And

$\mathrm{M}_{\gamma_{\mathrm{Rsel}}}(\mathrm{s})=\mathrm{M}\left(\begin{array}{c}\mathrm{M}-1 \\ 0\end{array}\right) \sum_{\mathrm{k}=0}^{\mathrm{M}-1}(-1)^{\mathrm{k}} \frac{1\left(\begin{array}{c}\mathrm{M}-1 \\ \mathrm{k}\end{array}\right)}{\mathrm{k}+1+\mathrm{s} \bar{\gamma}_{\mathrm{i}}}$

The BEP (Bit Error Probability) of system is obtained by substituting (11) and (12) into (10) and evaluating the integration with a similar way in [12], $P_{b}(e)$ can be obtained in a closed form as:

$$
\begin{gathered}
P_{b}=A \times M\left(\begin{array}{c}
M-1 \\
0
\end{array}\right) \sum_{k=0}^{M-1}(-1)^{k}\left(\begin{array}{c}
M-1 \\
k
\end{array}\right) \frac{1}{k+1} \\
\times\left(1-\frac{\bar{\gamma}_{i} /(2 k+2)}{\bar{\gamma}_{i} /(2 k+2)-\bar{\gamma}_{S, D}} \sqrt{\frac{B \bar{\gamma}_{i} /(2 k+2)}{1+B \bar{\gamma}_{i} /(2 k+2)}}\right. \\
\left.+\frac{\bar{\gamma}_{S, D}}{\bar{\gamma}_{i} /(2 k+2)-\bar{\gamma}_{S, D}} \sqrt{\frac{B \bar{\gamma}_{S, D}}{1+B \bar{\gamma}_{S, D}}}\right)
\end{gathered}
$$

where $\mathrm{A}$ is a parameter relating to the considered modulation, $\bar{\gamma}_{S, D}$ is average SNR between S-D and given as $\bar{\gamma}_{S, D}=\sqrt{P \xi} E\left(h_{S, D}^{2}\right) / N_{0}$. $\mathrm{E}($.$) is the statistical average (or$ expectation) operator. $\bar{\gamma}_{i}$ is average SNR and can be expressed in terms of the average SNR $\bar{\gamma}_{S, R_{i}}=\sqrt{P \xi} E\left(h_{S, R_{i}}^{2}\right) / N_{0}, \bar{\gamma}_{R_{i}, D}=\sqrt{P(1-\xi)} E\left(h_{R, D}^{2}\right) / N_{0}$. In addition $\bar{\gamma}_{i}$ defined as:

$\bar{\gamma}_{\mathrm{i}}=\frac{\bar{\gamma}_{\mathrm{S}, \mathrm{R}_{\mathrm{i}}} \bar{\gamma}_{\mathrm{R}_{\mathrm{i}} \mathrm{D}}}{\left(\bar{\gamma}_{\mathrm{S}, \mathrm{R}_{\mathrm{i}}}+\bar{\gamma}_{\mathrm{R}_{\mathrm{i}}, \mathrm{D}}\right)}$

In (13) $M$ represents the number of the available relays in the AF relay selection system.

\section{RESULTS}

In order to obtain optimal power allocation between source and the best relay, the BEP expression in (13) must be minimized. In other words, we have to find a $\xi$ value that minimizes the BEP at each SNR value. However, the optimum power ratio can't be found easily as a closed form expression. The BEP expression has be derivated with respect to $\xi$. The derivation of the BEP and then the solution of the equation are very complicated. To overcome of this problem, we first use a numerical method to obtain an optimum value of $\xi$. Here, the numerical method means that various values for $\xi$ are used at each SNR value, then the one that gives the minimum BEP is taken as an optimum value. The contribution of this work is the use of ANNs to obtain the optimum value for $\xi$ at each SNR value without trying all possible values of $\xi$.

In this section, we first calculate the minimum BEP results obtained by the numerical method, then compare with those obtained by the ANN method. Through this section, the considered modulation is BPSK in the AF relay selection scheme. The number of available relays is four (i.e., $M=4$ ) in all scenarios.
Power ratio values, give the minimum BEP at each SNR in case of the channel gains are unity, i.e., hsr=1, $\mathrm{hrd}=1$ and $\mathrm{hsd}=1$, are calculated between 0 and 1 by numerical and using ANN. It is seen in that ANN results are almost similar with the numerical results. The optimum values for the power ratio determined by the ANN versus Numeric are listed in Table I.

TABLE I

OPTIMUM VALUES FOR THE POWER RATIO(ANN VS NUMERIC)

\begin{tabular}{|c|c|c|c|c|c|c|}
\hline SNR(dB) & $\mathbf{0}$ & $\mathbf{5}$ & $\mathbf{1 0}$ & $\mathbf{1 5}$ & $\mathbf{2 0}$ & $\mathbf{2 5}$ \\
\hline $\begin{array}{c}\text { Optimum } \\
\text { Power } \\
\text { Ratio(ANN) }\end{array}$ & 0.6654 & 0.6093 & 0.5831 & 0.5703 & 0.5616 & 0.5519 \\
$\begin{array}{c}\text { Optimum } \\
\text { Power } \\
\text { Ratio(Numeric) }\end{array}$ & 0.6464 & 0.64 & 0.6332 & 0.6262 & 0.6191 & 0.6121 \\
\hline
\end{tabular}

Next, we investigate the BEP performances of AF Relay Selection Scheme under different channel gains. From Fig. 3 to Fig. 4, the optimum power ratio values are calculated at each SNR via the ANN model, and compared with the fixed values $(\xi=0.1,0.3,0.5,0.7$ and 0.9 ) for the power ratio. Fig. 3 shows the results in case of a high channel gain, i.e., 10 between $R$ and $D$ whereas the channel gains S-R and S-D are unity= In Fig. 4, it is considered that the channel quality is high for S-R, and the unity for the rest.

Finally, we investigate the effects of channel quality (or gain) on the optimum power ratio. From Fig. 5 to Fig. 6, we present the optimum power ratios versus $h_{R D}, h_{S R}$ respectively at a SNR value of $10 \mathrm{~dB}$. The optimum values shown in Fig. 5-6 are calculated by the ANN model and the numeric method.

It is observed in Fig. 5-6 that the optimum power ratio rises, when the channel gain between the relay and the destination is increasing. In contrast, when the channel gain between the source and the relay rises, the optimum power ratio reduces.

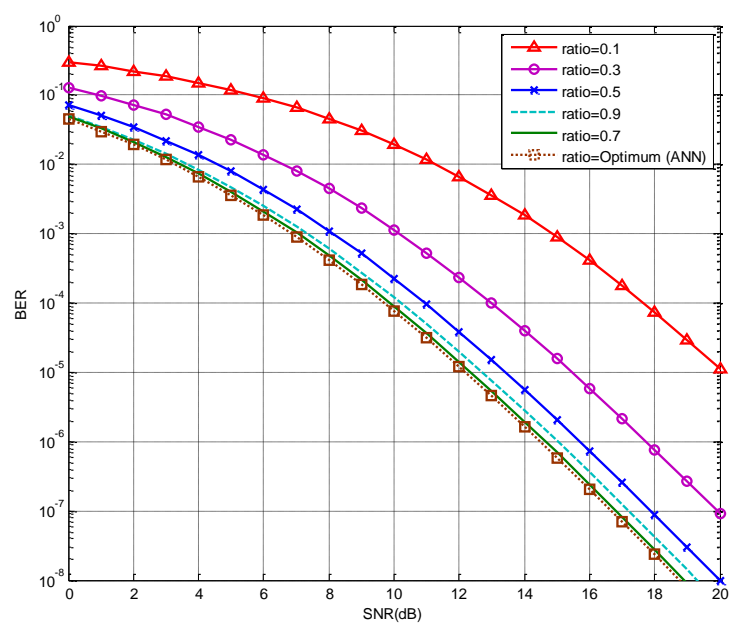

Fig. 3 The BEP performances for $h s r=1$, hrd=10 and $h s d=1$ 


\section{CONCLUSION}

In this study, the optimum power allocation in a cooperative communication system with relay selection over Rayleigh fading channels is investigated. Since the derivation of the optimum power ratio from probability density functions is very complicated, ANNs are utilized to obtain optimum power allocation values under different channel conditions. The use of ANNs reduces the mathematical complexity and simplifies the arithmetic equations. Once, an optimum power ratio between the total power and the transmitted power at source are found, the BEP performance using the optimum power ratio improves.

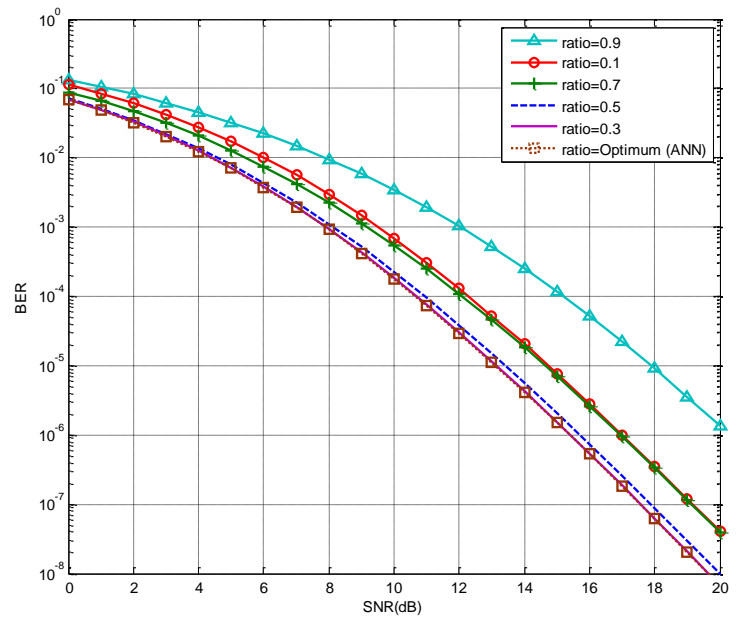

Fig. 4 The BEP performances for $h_{s r}=10, h_{r d}=1$ and $h_{s d}=1$

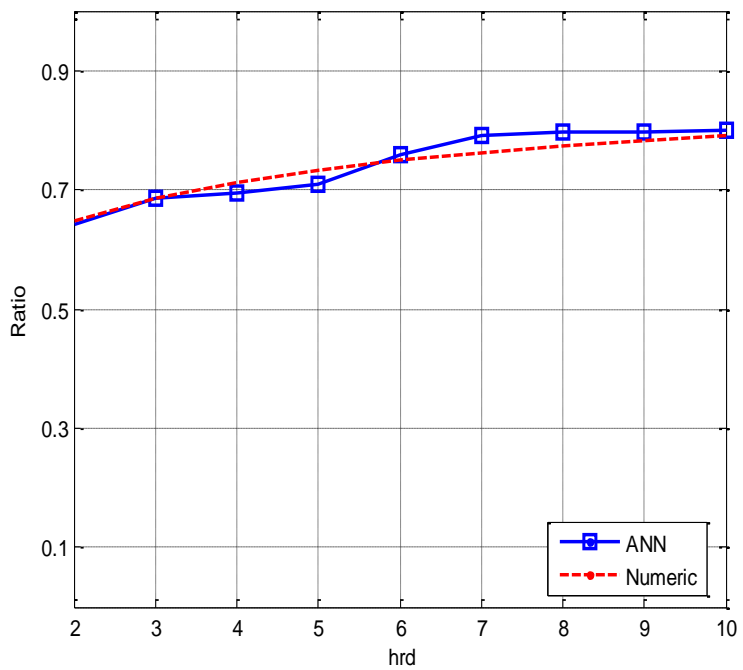

Fig. 5The optimum power ratios versus $h_{R D}$ for $S N R=10 d B$.

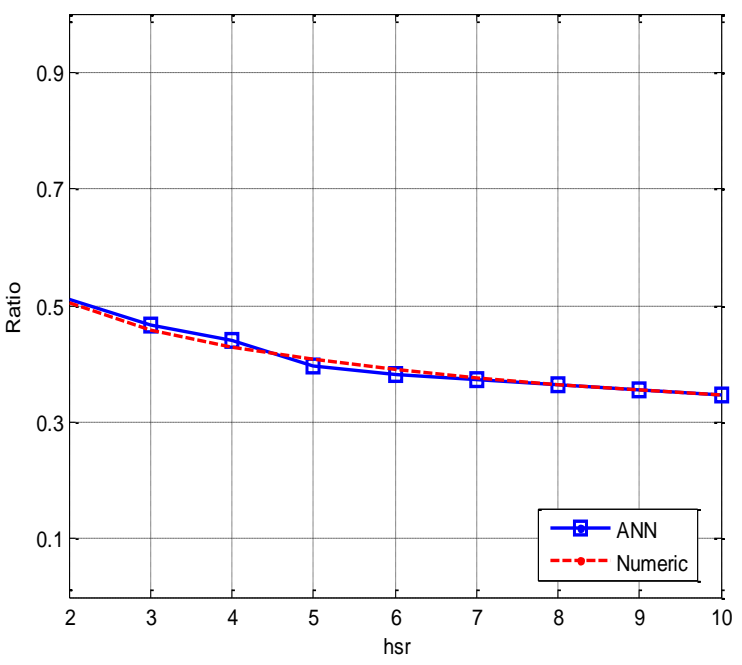

Fig. 6 The optimum power ratios versus $h_{S R}$ for $S N R=10 d B$.

\section{REFERENCES}

[1] S.Haykin , "Neural Networks: A Comprehensive Foundation", Newyork: Mcamillan and IEEE Computer Society, 1999.

[2] H. Zhang, Z. Wang, and D. Liu, "A comprehensive review of stability analysis of continuous-time recurrent neural networks", IEEE Trans.Neural Netw. Learn. Syst., vol. 25, no. 7, pp. 1229-1262, Jul. 2014

[3] Moustafa, M., El Ramly, S.H.A., "Channel estimation and equalization using backpropagation neural networks in OFDM systems", Wireless and Optical Communications Networks, 2009. WOCN '09. IFIP International Conference, ,Cairo, pp. 1-4 ,April 2009.

[4] Tao Cui, Tellambura,C. "Channel estimation for OFDM systems based on adaptive radial basis function networks" Vehicular Technology Conference, 2004. VTC2004-Fall. 2004 IEEE 60th, vol 1., pp. 608-611, Sept. 2004

[5] Xuantao Lyu, Wei Feng, Rui Shi, Yukui Pei,Ning Ge," Artificial neural network-based nonlinear channel equalization : A soft-outpu perspective", Telecommunications (ICT), 2015 22nd International Conference, Sydney, pp.243-248, April 2015

[6] Kassab, J. , Nagaraj, S. , "Adaptive Modulation in an OFDM Communications System with Artifical Neural Networks", Neural Networks, 2009. IJCNN 2009. International Joint Conference, Atlanta, pp.1547-1551, June 2009

[7] A. Goldsmith, Wireless Communications, Cambridge University Press., 2005.

[8] Sendonaris, A., Erkip, E., Aazhang, B., "User Cooperation DiversityPart I: System Description”, IEEE T. Commun., 51: 1927-1938, 2003.

[9] K. J. Ray Liu, Ahmed K. Sadek, Weifeng Su, Andres Kwasinski, Cooperative Communications and Networking, Cambridge University Press, 2009.

[10] Bletsas, A., Khisti, A., Reed, D.P., and Lippman, A., "A simple Cooperative diversity method based on network path selection." IEEE Journal on Selected Areas in Communications, 24(3):659-672, 2006.

[11] S. Ikki, M.H. Ahmed, "On the Performance of Amplify-and-Forward Cooperative Diversity with the $\mathrm{N}^{\text {th }}$ Best-Relay Selection Scheme ",Communications, 2009. ICC '09. IEEE International Conference, Dresden, pp. 1-6, June 2009.

[12] H.S. Efendioglu, T. Yildirim, and K. Fidanboylu, "Prediction of Force Measurements of a Microbend Sensor Based on an Artificial Neural Network", Sensors, Vol. 9, No. 9, 7167-7176, 2009.

[13] Jia Wang, Xian-Ming Zhang, and Qing-Long Han,” Event-Triggered Generalized Dissipativity Filtering for Neural Networks With TimeVarying Delays", IEEE Trans.Neural Netw. Learn. Syst., vol. 27, no:1, pp.77-78, Jan.2016. 